\title{
Controlling interfacial interactions in LDPE/flax fibre biocomposites by a combined chemical and radiation-induced grafting approach
}

\author{
Youssef EI Moussi - Belkacem Otazaghine $\cdot$ Anne-Sophie Caro-Bretelle $\cdot$ Rodolphe Sonnier $\cdot$ Aurélie \\ Taguet $\cdot$ Nicolas Le Moigne
}

\begin{abstract}
By combining chemical and gamma radiation treatments, we show that it is possible to improve the interfacial adhesion between natural fibres and a non-polar and non-reactive matrix such as low density polyethylene (LDPE), and the resulting mechanical performances of their biocomposites. To this aim, flax fibres are first functionalized with octadecylphosphonic acid (ODPA) and the quantity, effective grafting and localization of ODPA molecules on flax fibres are characterized by ICP-AES, solid state ${ }^{31} \mathrm{P}$ NMR and SEM-EDX. ODPA molecules are effectively grafted onto flax fibres at a content of roughly $2.5 \mathrm{wt} \%$ and mostly localized at the fibre surface. Moreover,
\end{abstract}

IMT Mines Alès is member of the European Polysaccharide Network of Excellence (EPNOE), http://www.epnoe.eu. contact angle measurements as well as water sorption kinetics give evidence for increased hydrophobic and oleophilic character of ODPA treated fibres. Pellets of LDPE/flax fibre biocomposites obtained by melt extrusion are then submitted to gamma radiation and processed by injection moulding. Based on Soxhlet extraction experiments and SEM observations, it is shown that LDPE cross-linking rate induced by gamma radiation is locally enhanced at the fibre/matrix interface, suggesting that regio-selective crosslinking occurred between LDPE chains and the alkyl chain of ODPA grafted on flax fibre surface. Consequently, the uniaxial tensile performances of the biocomposites are enhanced by this combined chemical and radiation-induced grafting approach, especially their ultimate properties (up to $40 \%$ increase in the work of rupture). These results evidence an enhanced interfacial adhesion that is also supported by more cohesive interfacial failure and higher work of rupture through in-situ micro-mechanical tensile SEM experiments.

Y. El Moussi · B. Otazaghine · R. Sonnier ·

A. Taguet $(\square) \cdot$ N. Le Moigne $(\square)$

Polymers Composites and Hybrids (PCH) - IMT Mines

Ales, Ales, France

e-mail: aurelie.taguet@mines-ales.fr

N. Le Moigne

e-mail: nicolas.le-moigne@mines-ales.fr

A.-S. Caro-Bretelle

LMGC, IMT Mines Ales, Univ Montpellier, CNRS, Ales, France 


\section{Graphic abstract}

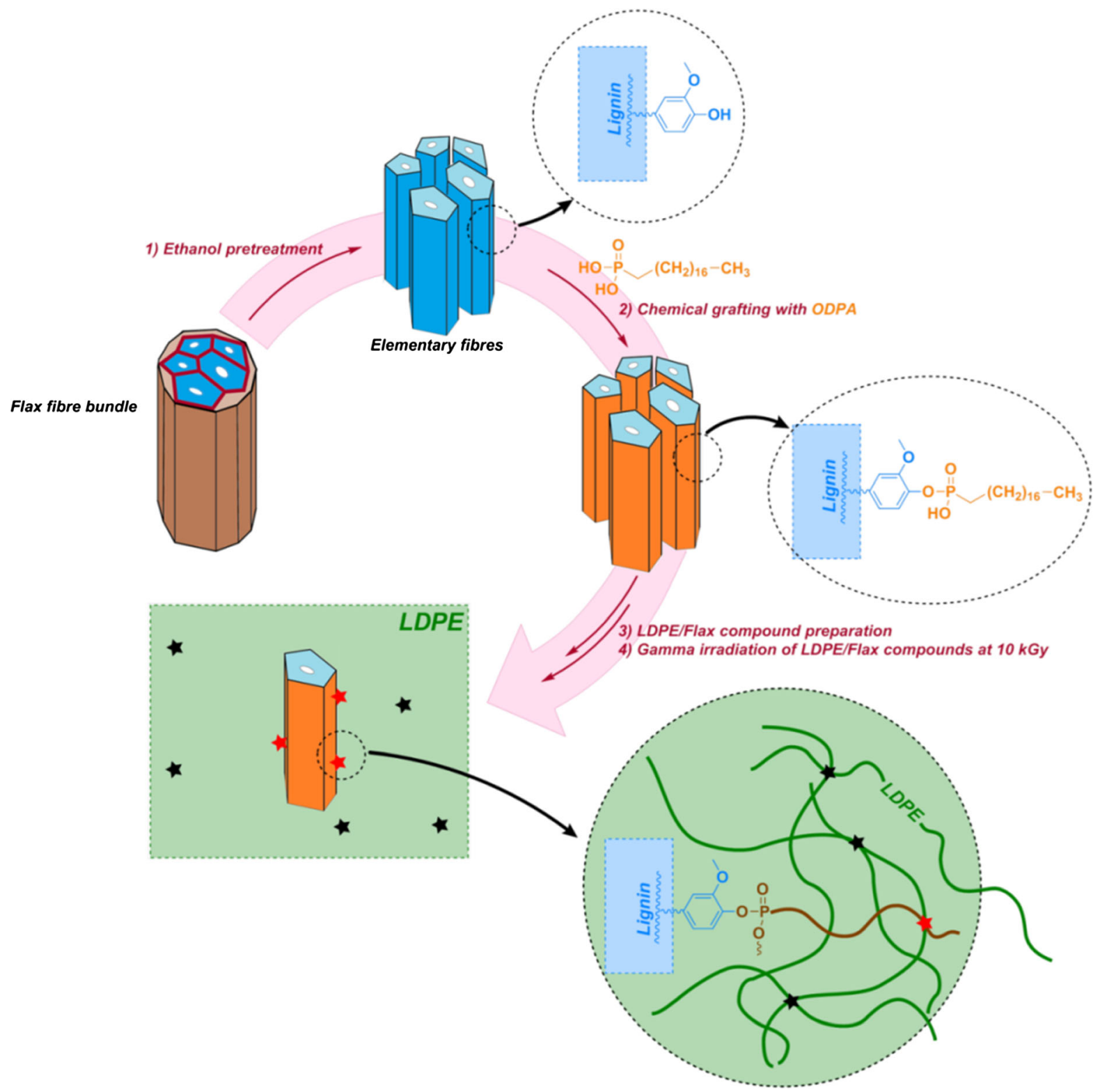

Keywords Biocomposite - Flax fibres - Interface · Grafting $\cdot$ Radiation

\section{Introduction}

Current environmental issues and related societal concerns towards a better used of natural resources in materials applications make natural fibres promising alternative reinforcements for composite applications (Müssig 2010; Yan et al. 2014; Bourmaud et al. 2018). Natural fibres have several advantages compared to synthetic fibres such as glass, in particular their biobased and renewable origin and biodegradability, low cost, low density, low abrasiveness and their excellent damping and specific mechanical 
performance. Nevertheless, the use of natural fibres in composite applications is limited to polymer matrices of low processing temperatures (i.e. polyolefins, biopolyesters and some bio-based polyamides) due to their low thermal stability.

Beyond intrinsic properties of natural fibres and polymer matrices, interfacial adhesion in between plays a key role in stress transfer taking place at the fibre/matrix interface, as well as a protective role towards ageing. In this regard, mechanical performances of biocomposites are limited by the weak (physico-)chemical interactions at the fibre/matrix interface. The quality and strength of the interfacial adhesion is primarily related to surface energy and work of adhesion between polymers and fibres, the fibre surface topography, as well as the presence of functional molecules at the fibre surfaces that can react or entangle with polymer chains of the matrix (Le Moigne et al. 2018a). It should be pointed out that internal cohesion between the cell wall layers of natural fibres as well as the presence of weakly bonded components at their surface are also decisive for the strength of interfacial adhesion within natural fibres/ polymer systems (Le Duigou et al. 2012; Le Moigne et al. 2014; Moigne et al. 2018b). Other parameters should also be considered as the hygroscopic radial expansion of natural fibres due to moisture that could play a positive role on load transfer and interfacial adhesion if radial stresses are controlled and do not induce damage within the composites microstructure (Le Duigou et al. 2017; Réquilé et al. 2019).

In this context, natural fibre surface modifications are a major strategy for improving interfacial adhesion with polymer matrices. Thereby, active research is ongoing on the development of efficient chemical and physical treatment methods and functionalization of natural fibres aiming to improve biocomposite mechanical performances (Le Moigne et al. 2018c). In the case of non-polar and non-reactive matrix such as polyolefins, a classical approach used at the industrial scale is the incorporation of maleated polymers (MAPP, MAPE) during extrusion that react with hydroxyl groups of natural fibres by condensation reactions and interact with the matrix by physical entanglements (Kazayawoko et al. 1999; Xie et al. 2010). The main limitation of this approach is the diffusion capacity of the maleated polymer chains at the fibre/matrix interface (Le Moigne et al. 2018c) and the limited cohesion brought by physical entanglements. Esterification of various ligno-cellulosic fibres (flax, sisal, wood pulp) with saturated and unsaturated fatty acids, as stearic acid and various fatty acyl chlorides, has been also considered in order to increase interfacial shear strength (IFSS) between fibres and polyolefins (Zafeiropoulos et al. 2002; Torres and Cubillas 2005; Freire et al. 2006, 2008; Dogan et al. 2016). Substantial improvements in interfacial adhesion were achieved although degradation of fibres and the decrease of their thermal stability were reported in some studies. Besides, these fibres functionalization approaches primarily aim to improve physico-chemical interfacial interactions through an increase of the work of adhesion. Indeed, no chemical grafting between the matrix and the functionalizing molecules at the fibre surface occurs. In this regard, natural fibres have been also treated with organosilanes such as MPS ( $\gamma$-methacryloxypropyl trialkoxy silane), VTS (vinyl trialkoxy silane) and VTMO (vinyl trimethoxy silane) in combination with peroxides (e.g. dicumyl peroxide DCP) through reactive extrusion. The aim of this strategy is to induce a free radical grafting between polyethylene (PE) or polypropylene (PP) chains and the $\mathrm{C}=\mathrm{C}$ bonds beared by the organosilanes grafted at the fibre surfaces (Xie et al. 2010). However, peroxides also induce self-cross-linking of the matrix and could result in a significant decrease of its ductility which could be detrimental to impact strength of the composites.

We propose an innovative approach combining chemical and radiation-induced grafting so as to modify interfacial interactions in low density polyethylene (LDPE)/flax biocomposites and create covalent bonds on both fibre and matrix sides. As reviewed by Le Moigne et al. (2017), ionizing radiation methods can be relevant for the surface modification and functionalization of natural fibres as reinforcements in composite applications. A suitable range of radiation doses $(<30 \mathrm{kGy})$ allows radiation-grafting of molecules onto natural fibres while preserving their structural integrity and physical properties. Furthermore, high grafting yields can be reached quickly without heating and solvents. In the present study, flax fibres are functionalized with octadecylphosphonic acid (ODPA) (Dorez et al. 2014) before their introduction in LDPE matrix. Pellets of biocomposites obtained by melt extrusion are submitted to gamma irradiation before being injection molded. The influence of the 
combined chemical and radiation-induced grafting treatments on the microstructure, tensile mechanical behaviour and fibre/matrix interfacial adhesion of biocomposites is investigated.

\section{Material and methods}

\section{Materials}

The untreated flax fibres of average length $2.24 \mathrm{~mm}$ and width $110 \mu \mathrm{m}$ were supplied by Fibres Recherche Développement (Troyes, France). Their biochemical composition was characterized according to Van Soest method (standards NF EN ISO 13,906 and NF V18122): cellulose $(82.8 \pm 0.4 \mathrm{wt} \%)$, hemicelluloses $(5.4 \pm 0.4 \mathrm{wt} \%)$, lignin $(1.7 \pm 0.1 \mathrm{wt} \%)$, solubles $(9.7 \pm 0.7 \mathrm{wt} \%)$ and ashes $(0.4 \pm 0.1 \mathrm{wt} \%)$. Low density polyethylene (LDPE) provided by Repsol AlcudiaPE017 (Spain), was used in this study, and has a density of $0.919 \mathrm{~g} / \mathrm{cm}^{3}$ and melting temperature of $108^{\circ} \mathrm{C}$.

Octadecylphosphonic acid (ODPA) was supplied by ABCR (Germany) and chosen for its reactivity towards hydroxyl groups of flax fibres. Absolute ethanol was provided by Fisher Scientific (Waltham MA, USA). All products were used as received without any further purification.

\section{Chemical treatment of flax fibres}

The treatment of flax fibres with ODPA was achieved in two steps according to the procedure developed in (Dorez et al. 2014). First, the flax fibres were pretreated with ethanol to remove waxes from the surface of the fibres and ease the separation of fibre bundles into elementary fibres (Acera Fernández et al. 2016). This pre-treatment should also promote the grafting of ODPA molecules onto elementary flax fibres by removal of extractives which could react with ODPA. For the pre-treatment, about $10 \mathrm{~g}$ of fibres and $200 \mathrm{ml}$ of a (90/10 wt $\%)$ ethanol/demineralized water mixture were introduced into a one-necked round bottom flask (250 $\mathrm{ml}$ ) equipped with a condenser. The mixture was heated at $100{ }^{\circ} \mathrm{C}$ under reflux for $2 \mathrm{~h}$ with magnetic stirring. The liquid phase was removed, then fibres were washed twice with absolute ethanol and dried under a ventilated hood at room temperature during $48 \mathrm{~h}$. The same reflux system was used to achieve the functionalization step with ODPA. The procedure was the following: around $10 \mathrm{~g}$ of pre-treated flax fibres were introduced into an ethanol/demineralized water (90/10 wt \%) solution containing $5 \mathrm{wt} \%$ (based on fibre weight) of ODPA. The mixture was then heated at $100{ }^{\circ} \mathrm{C}$ under reflux for $4 \mathrm{~h}$. The treated fibres were washed twice with ethanol to remove unreacted ODPA molecules, then filtered and dried under a ventilated hood at room temperature until the evaporation of ethanol was completed (Dorez et al. 2014). The designations of the untreated, ethanol pre-treated and ODPA treated flax fibres are respectively: Flax-NT, Flax-PT and Flax-T.

Preparation of LDPE/flax fibre biocomposites

Blends of LDPE filled with $20 \mathrm{wt} \%$ of untreated or treated flax fibres were compounded using a twinscrew micro-extruder (DSM Xplore) at $180{ }^{\circ} \mathrm{C}$, with a screw speed of $60 \mathrm{rpm}$. The residence time in the microcompounder was roughly $4 \mathrm{~min}$. Prior to compounding, LDPE matrix and flax fibres were dried at $60{ }^{\circ} \mathrm{C}$ under vacuum overnight to remove residual water. After pelletizing and drying at $80{ }^{\circ} \mathrm{C}$ under vacuum for $24 \mathrm{~h}$, compounds were injection-molded using a small injection moulding device Zamak Mercator, to obtain testing specimens (ISO 1BA) for the measurement of uniaxial tensile properties. During injection, pellets were heated at $180{ }^{\circ} \mathrm{C}$ for $80 \mathrm{~s}$ in the heating chamber. Melted pellets were maintained at 3 bars during $10 \mathrm{~s}$ in the mould held at a constant temperature of $25^{\circ} \mathrm{C}$. The designations of the untreated and ODPA treated biocomposites samples are LDPE/Flax_NT and LDPE/Flax_T, respectively.

Gamma radiation treatment of LDPE/flax fibre biocomposites

Prior to injection moulding, pellets of biocomposites were irradiated under air at room temperature by Ionisos (Dagneux, France) using a ${ }^{60} \mathrm{Co}$ source. The gamma radiation dose was set at $10 \mathrm{kGy}$, with an irradiation rate of $2 \mathrm{kGy} / \mathrm{h}$ in order to avoid fibre degradation (Le Moigne et al. 2017) and limit PE selfcross-linking (Ferreto et al. 2012; Alvarez and Perez 2013). The designations of the non-irradiated and irradiated biocomposite samples are LDPE/Flax_NI and LDPE/Flax_I, respectively. Neat LDPE was also irradiated in the same conditions as a reference 
(LDPE_I). Based on literature related to radiation effects in polyethylene and $n$-alkanes (Ungar 1981), Fig. 1 presents the expected reaction scheme between ODPA grafted flax fibres and LDPE matrix upon gamma radiation. Two mechanisms are likely to occur upon radiation and formation of free radicals: (i) ODPA-LDPE radical reaction that should contribute to improve fibre/matrix interfacial adhesion and (ii) LDPE self-cross-linking that could modify crystallization and mechanical behaviour of LDPE.

Characterization of flax fibres

\section{Inductively coupled plasma atomic emission spectroscopy (ICP-AES)}

The content of phosphorus in treated fibres was determined by Inductively Coupled Plasma Atomic Emission Spectroscopy (ICP-AES). This elemental analysis consisted of several steps: first, $50 \mathrm{mg}$ of untreated or ODPA treated flax fibres were dissolved in a mixture of strong acids containing $1 \mathrm{ml}$ of nitric acid $(63 \%)$ and $2 \mathrm{ml}$ of sulfuric acid (98\%). Then the preparation was heated by microwaves in the power range $400-700 \mathrm{~W}$. The resulting mixture was diluted with demineralized water until reaching $50 \mathrm{ml}$, and finally introduced into the plasma set at $6000{ }^{\circ} \mathrm{C}$. This thermal excitation causes a phenomenon of ionization and separation of the elements. A Thermo Finnigan Flash EA 112 Series was used for the elemental analysis of phosphorus. Each sample was analysed three times. Eq. (1) was used to calculate the content of ODPA grafted on treated fibres:
$C_{O D P A}=\left(\frac{C_{f}(P)-C_{i}(P)}{\frac{M(P)}{M(O D P A)}}\right)$

where $C_{f}(P)$ is the content of phosphorus in treated fibres (wt\%), $C_{i}(P)$ is the content of phosphorus in untreated fibres $(\%), M(P)$ is the molar mass of phosphorus $(30.97 \mathrm{~g} / \mathrm{mol})$ and $M(O D P A)$ is the molar mass of ODPA $(334.47 \mathrm{~g} / \mathrm{mol})$.

\section{Solid state ${ }^{31} P$ NMR (nuclear magnetic resonance) spectroscopy}

These experiments were carried out by the IRCELYON (Lyon, France). The ${ }^{31} \mathrm{P}$ NMR spectra were taken with an AVANCE III 500WB spectrometer equipped with a probe of $4 \mathrm{~mm}$ triple resonance $\mathrm{HXY}$, operating at a frequency of $202.41 \mathrm{MHz}$. The chemical shift of ${ }^{31} \mathrm{P}$ was externally referenced to the phosphorus of $\mathrm{H}_{3} \mathrm{PO}_{4} 85 \mathrm{wt} \%$. The ${ }^{31} \mathrm{P}$ MAS NMR spectra was recorded using these experimental conditions: CP-MAS magic angle spinning, $90^{\circ}$ pulses, rotation speed of $10 \mathrm{kHz}$, pulse acquisition time of $3.25 \mu \mathrm{s}$, spectral width of $300 \mathrm{ppm}$, recycle delay of $40 \mathrm{~s}$. About 5770 acquisitions were required to obtain an adequate signal for each sample.

Scanning electron microscopy (SEM) coupled energy dispersive $X$-ray $(E D X)$ analysis

Untreated and ODPA treated flax fibres were observed by an environmental scanning electron microscope (FEI Quanta 200) equipped with a XMAX $80 \mathrm{~mm}^{2}$ Oxford Instrument detector. The samples were pasted
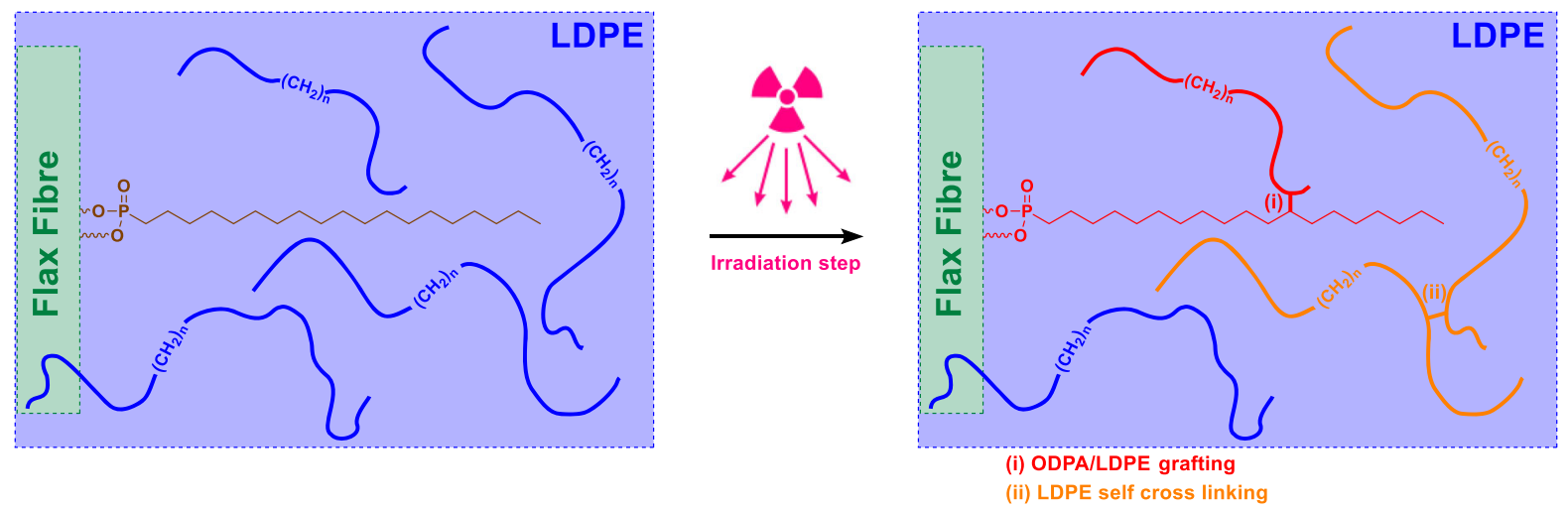

Fig. 1 Expected reaction scheme between ODPA grafted flax fibres and LDPE matrix upon gamma radiation: (i) ODPA-LDPE radical reaction and (ii) LDPE self-cross-linking 
on an adhesive wafer and metallized in high vacuum by depositing a layer of carbon on the surface using a BALZERS CED 030 in order to avoid any degradation during analysis. Micrographs were obtained under high vacuum at an acceleration voltage between 10 and $12.5 \mathrm{keV}$, a pressure between 0.8 and 0.9 Torr and a working distance between 9 and $16 \mathrm{~mm}$. The elemental analysis and cartographies of phosphorus on the cross-sections of flax fibres were carried out using energy dispersive X-ray spectroscopy in order to locate phosphorus and hence ODPA molecules present in the fibres.

\section{Contact angle measurements}

The wettability of flax fibres with water and diiodomethane was determined with a KRUSSDSA30 goniometer apparatus driven by KRÜSS ADVANCE software and equipped with a camera working at up to 50 images per second. About $0.4 \mathrm{mg}$ of untreated or ODPA treated flax fibres were compressed into disks at room temperature using a cylindrical steel mould with an applied pressure of about 20 bars for $3 \mathrm{~min}$. At least five droplets of $3 \mu \mathrm{l}$ of water and $1 \mu \mathrm{l}$ of diiodomethane were deposited on the surface of compressed fibre disks. Average initial contact angles were obtained in air at $23{ }^{\circ} \mathrm{C}$ and taken less than $1 \mathrm{~s}$ after droplet deposition. In addition, water sorption kinetics into the samples in air at $23{ }^{\circ} \mathrm{C}$ was analysed by monitoring water contact angles over $10 \mathrm{~min}$.

\section{Characterization of LDPE/flax fibre biocomposites}

\section{Soxhlet extraction}

Gel fraction was measured by Soxhlet extraction in order to quantify the insoluble fraction of LDPE (i.e. crosslinked LDPE) after gamma irradiation. Samples of the neat matrix and different biocomposites ( $\sim 200 \mathrm{mg}$ ) cut from injection moulded specimens were put in a Soxhlet extractor for $48 \mathrm{~h}$ using boiling xylene (at $140{ }^{\circ} \mathrm{C}$ ) as solvent. Experiments were done at least in duplicate. The soluble fraction of LDPE was completely removed during Soxhlet, whereas the solid residue which contained flax fibres and cross-linked LDPE chains was air-dried until a constant weight was reached. Soxhlet residues were also observed by SEM as detailed in Sect. 2.6.4. Eq. (2) was used to calculate LDPE gel fraction:

$\operatorname{Gelfraction}(\%)=\frac{\left(m_{\text {residue }}-m_{\text {fibres }}\right)}{m_{L D P E}} \times 100$

where $m_{\text {residue }}(\mathrm{mg})$ is the weight of the solid residue after Soxhlet extraction, $m_{L D P E}(\mathrm{mg})$ is the initial weight of LDPE and $m_{\text {fibres }}(\mathrm{mg})$ is the initial weight of flax fibres within the biocomposites, i.e. $80 \mathrm{wt} \%$ and $20 \mathrm{wt} \%$ of the total initial sample weight, respectively.

\section{Differential scanning calorimetry (DSC)}

Perkin-Elmer Pyris Diamond DSC was used to determine the degree of crystallinity of biocomposites $\left(X_{c}\right)$. Samples of $10 \mathrm{mg}$ cut from the injection moulded specimens, were placed in aluminum pans, and first heated from 10 to $180{ }^{\circ} \mathrm{C}$, then cooled down to $10{ }^{\circ} \mathrm{C}$ and finally heated again up to $180{ }^{\circ} \mathrm{C}$ at heating/cooling rates of $10{ }^{\circ} \mathrm{C} / \mathrm{min}$ and holding times of $3 \mathrm{~min}$ between each heating/cooling step. Melting enthalpies were determined between 40 and $115^{\circ} \mathrm{C}$ on the first heating ramp $\left(\Delta \mathrm{H}_{\mathrm{m} 1}\right)$. Two samples were tested per formulation. The degree of crystallinity $X_{c}$ was determined according to the Eq. (3):

$\left.X_{c}(\%)=\frac{\left|\Delta H_{m 1}\right|}{\left|\Delta H_{m}^{0}\right| \times W_{L D P E}}\right) \times 100$

where $\Delta H_{m l}$ is the melting enthalpy of the sample $(\mathrm{J} /$ g), $\Delta H_{m}{ }^{0}$ is the melting enthalpy of $100 \%$ crystalline LDPE (293 J/g) (Lu and Sue 2002; Krishnaswamy and Yang 2007; Poltimäe et al. 2009) and $W_{L D P E}$ is the weight fraction of LDPE within the sample, i.e. 80 $\mathrm{wt} \%$.

\section{Uniaxial tensile properties}

Tensile mechanical behaviour of the biocomposites was investigated with a Zwick Roell TH010 instrument equipped with a force sensor of $2.5 \mathrm{kN}$ and a Zwick "Clip-on" extensometer to evaluate the Young's modulus. According to ISO 527 standard, the crosshead speed was $1 \mathrm{~mm} / \mathrm{min}$ and $20 \mathrm{~mm} / \mathrm{min}$ for the measurement of the Young's modulus and the ultimate tensile strength and strain, respectively. Young's modulus was determined between 0.05 and $0.25 \%$ strain. The tests were conducted at $20{ }^{\circ} \mathrm{C}$ and 
$50 \%$ relative humidity. Five replicates were done for each formulation.

\section{SEM observations and image analysis}

For each biocomposite, interfacial interactions between LDPE and flax fibres were analysed by SEM (FEI Quanta 200) observations of the solid residues after Soxhlet extraction and the failure surfaces after tensile tests. Microstructural analysis of the biocomposites was also conducted on tensile specimens polished in the central zone down to $300 \mu \mathrm{m}$ below the surface, ensuring that samples were observed in the shell/core zone (Bay and Tucker 1992; Abdennadher 2015). These polished samples were observed by SEM and cartographies of $3.5 \mathrm{~mm} \times 3.5 \mathrm{~mm}$ consisting of 16 SEM images were built using the software Aztec $₫$ (Oxford Instruments). All the SEM observations were made on sputter coated samples with carbon using a BALZERS CED 030 in order to avoid any degradation. Micrographs were obtained under high vacuum at acceleration voltages ranging from 10 to $12.5 \mathrm{keV}$.

Based on cartographies, fibre size and shape distributions were analysed with the software AphelionTM V.4.3.2 (ADCIS and Amerinex Applied Imaging Inc.), which automatically detects the outlines of the fibres present on the pictures. Fibre length (L) and width (W) were determined as the maximum and minimum Ferret diameters, respectively of about 1000 fibres per cartography. The fibre shape is defined by its aspect ratio $(\mathrm{L} / \mathrm{W})$, i.e. the ratio between the maximum and the minimum Ferret diameter. Overlapped fibres were excluded from the counts. Because of the pictures resolution $(0.66 \mu \mathrm{m} / \mathrm{pixel})$, the particles with the maximum size smaller than $18 \mu \mathrm{m}$ were not considered in the fibre size and shape distributions.

\section{In-situ micro-mechanical tensile SEM experiments}

The SEM was equipped with a tensile apparatus (DEBEN microtest, maximum load $5 \mathrm{kN}$ ) to analyse interfacial failure mechanisms. Direct observations of the crack propagation were conducted at a displacement speed of $0.2 \mathrm{~mm} / \mathrm{min}$ on notched specimens with dimensions of $20 \mathrm{~mm} \times 5 \mathrm{~mm} \times 2 \mathrm{~mm}$ and a notch of $2 \mathrm{~mm}$ depth and $45 \pm 1^{\circ}$ opening. It should be pointed out that sample preparation and surface polishing are essential to remove the $100 \mu \mathrm{m}$ thick polymer layer from the surface and better observe failure mechanisms between the fibres and the matrix. Experiments were reproduced twice per each sample.

\section{Results and discussion}

Characterization of untreated and treated flax fibres

\section{ODPA content and grafting onto flax fibres}

Phosphorus content in untreated flax fibres and ODPA treated fibres was measured using ICP-AES analysis. The content of phosphorus greatly increases after functionalization of flax fibres with ODPA, from 0.07 to $0.3 \mathrm{wt} \%$. These results confirm the presence of ODPA in treated fibres even after ethanol washing. According to Eq. (1), the quantity of ODPA in flax fibres after treatment reaches up to $2.48 \mathrm{wt} \%$.

${ }^{31} \mathrm{P}$ solid state NMR analysis was conducted to confirm the grafting of ODPA onto flax fibres during the chemical treatment. ${ }^{31} \mathrm{P}$ chemical shifts are sensitive to variations in the $\mathrm{O}-\mathrm{P}-\mathrm{O}$ bond angle as well as to the electronegativity of the nearest atoms. In Fig. 2, it is observed that the O-P-O peak of pure ODPA centered at $32 \mathrm{ppm}$ [data shown in Dorez et al. (2014)] is shifted towards a smaller displacement $(<25 \mathrm{ppm})$ and splitted in two peaks (22.3 and $20.5 \mathrm{ppm})$ in the case of ODPA treated fibres, indicating a strong interaction between the ODPA headgroups and flax fibres. The ${ }^{31} \mathrm{P}$ chemical shift observed in this work confirms the covalent bonding between ODPA and hydroxyl groups of flax fibres through the formation of phosphonate covalent bonds as shown for several substrates by ${ }^{31} \mathrm{P}$ solid state NMR

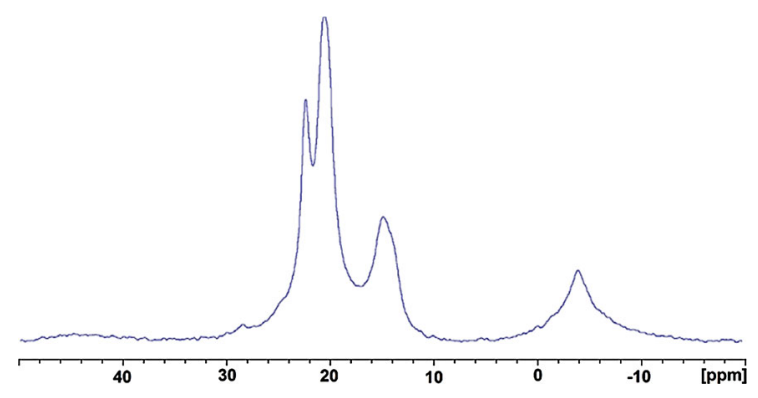

Fig. 2 Solid state ${ }^{31} \mathrm{P}$ NMR spectra of the ODPA treated flax fibres 
(Holland et al. 2007; Yah et al. 2012). It should be noticed that Dorez et al. (2014) showed by Py-GC/MS and PCFC experiments the preferential reaction of phosphonic acid moiety from ODPA with aromatic hydroxyl groups beared by lignin.

\section{Localization of ODPA in treated flax fibres}

Phosphorus mapping by SEM-EDX allows analysing the localization of ODPA on the treated fibres (Fig. 3). As already found in previous work (Dorez et al. 2014), phosphorus and hence ODPA is located into the whole cross-section of treated fibre bundles but its concentration is higher at the elementary fibre surfaces (Fig. 3b, d). This gradient of ODPA concentration highlights the limited diffusion of ODPA molecules through flax cell wall layers. This should be related to flax biochemical composition and reactivity of flax components towards ODPA molecules, as well as the chemical treatment conditions. It was shown previously using SEM-EDX that depending on the affinity between the solvent, the functionalizing molecule and the fibres, some phosphorus molecules can be grafted into the bulk of flax fibres (Sonnier et al. 2015; Hajj et al. 2018) whereas for other molecules their diffusion through flax cell wall layers is limited and they are only grafted at the elementary fibre surfaces (Sonnier et al. 2015).

Besides, it seems that the chemical (pre-)treatment promotes elementary fibre separation. Micrographs in Fig. 4 shows that elementary flax fibres in untreated fibres are packed into bundles (Fig. 4a) due to polysaccharide cements present in the middle lamellae (mainly pectins in the case of flax), whereas elementary flax fibres are well separated after the ethanol (pre-)treatment (Fig. 4b). In addition, surface components [containing mostly waxes (Marques et al. 2010) and pectins (Morvan et al. 2003)] were present on untreated fibre bundles and almost disappeared after (pre-)treatment, fibre surfaces being cleaner (Fig. 4b).
Fig. 3 SEM images of ODPA treated flax fibres (a) cross-sectional view; (c) surface view and corresponding phosphorus mapping of flax fibres $(\mathbf{b}, \mathbf{d})$
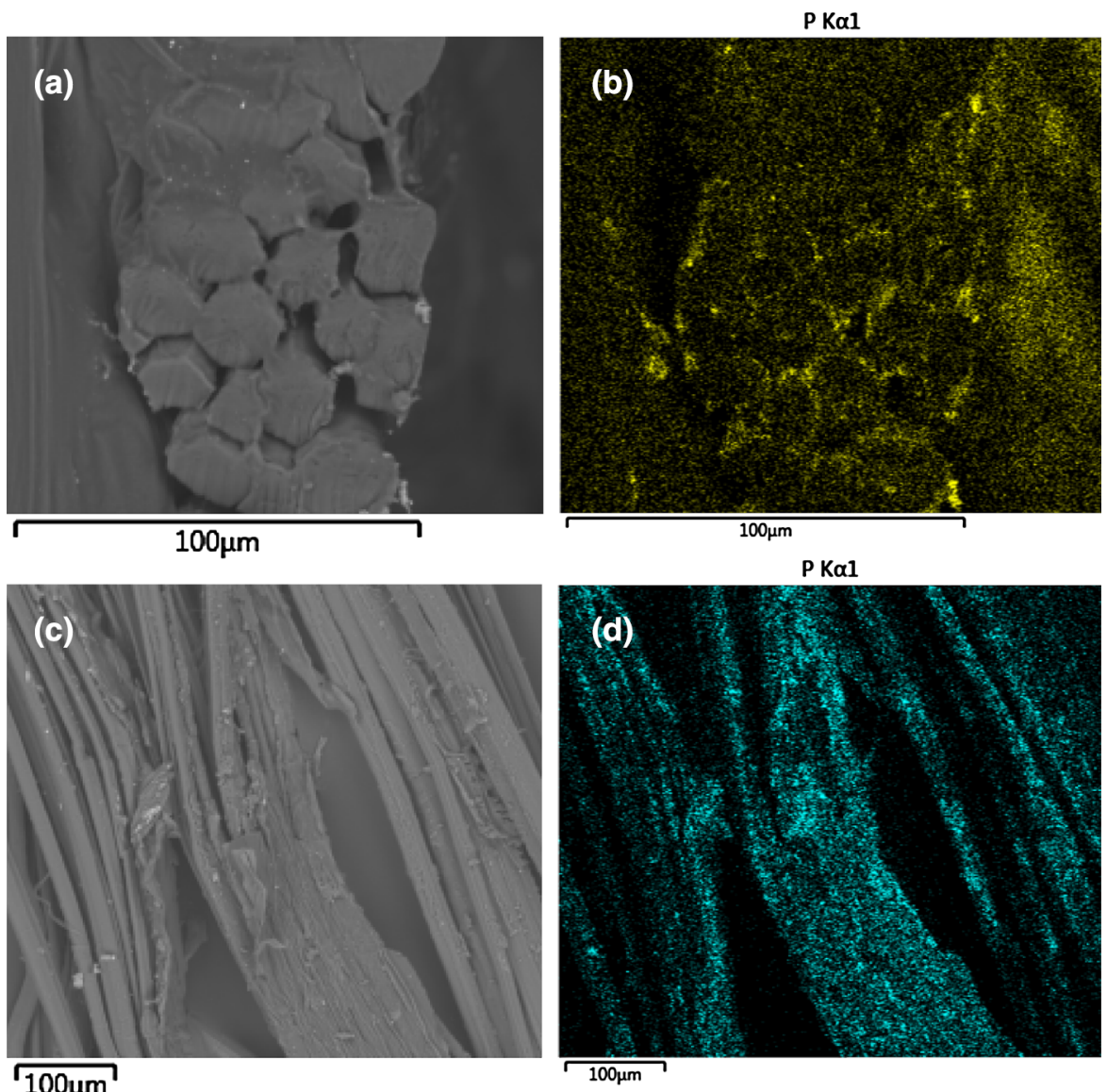
Fig. 4 SEM Micrographs of a untreated flax fibres, b ODPA treated flax fibres
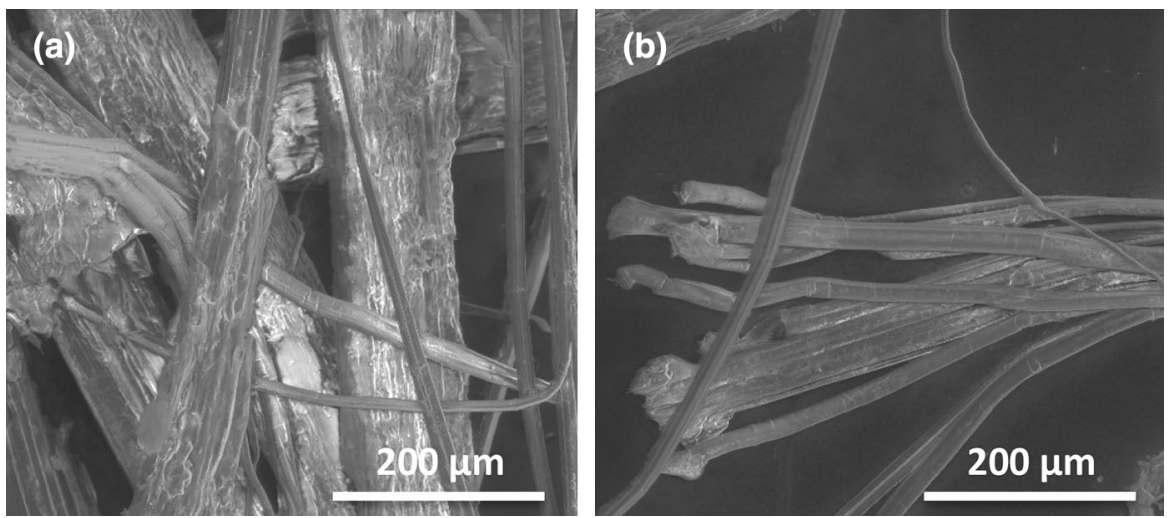

Better fibre individualization was thus achieved by the chemical (pre-)treatment which should promote higher specific interfacial area and hence improve interfacial adhesion (Coroller et al. 2013; Acera Fernández et al. 2016; Le Moigne et al. 2018a).

\section{Contact angle and water sorption kinetics in ODPA treated flax fibres}

Changes in hydrophilic/hydrophobic character of untreated, ethanol pre-treated and ODPA treated flax fibres were analysed by measuring contact angles with polar and non-polar liquids, i.e. water and diiodomethane. According to the results presented in Table 1, the initial water contact angle for untreated flax fibres is about $128.8^{\circ}$ whereas for ODPA treated flax fibres, the initial water contact angle is about $132.0^{\circ}$. Besides, the initial contact angle values with diiodomethane for the untreated flax fibres and ODPA treated fibres are $93.9^{\circ}$ and $88.6^{\circ}$, respectively. Moreover, the water contact angle was monitored over a period of $10 \mathrm{~min}$ (Fig. 5). Generally speaking, the water contact angle decreases gradually as the water is absorbed by the substrate and the volume of the water droplet

Table 1 Initial contact angles of untreated (Flax_NT), ethanol pre-treated (Flax_PT) and ODPA (Flax_T) treated flax fibres with water and diiodomethane

\begin{tabular}{lrl}
\hline & \multicolumn{2}{l}{ Contact angle $\left(\theta^{\circ}\right)$} \\
\cline { 2 - 3 } & \multicolumn{1}{l}{ Water } & Diiodomethane \\
\hline Flax_NT & $128.8 \pm 4.3$ & $93.9 \pm 15.6$ \\
Flax_PT & $81.3 \pm 9.2$ & - \\
Flax_T & $132.0 \pm 5.2$ & $88.6 \pm 13.2$ \\
\hline
\end{tabular}

decreases. Figure 5 clearly shows that water sorption kinetics is much slower after ODPA treatment, the water contact angle being more stable over time.

Initial contact angles with water and diiodomethane as well as water sorption kinetics give evidence for the increased hydrophobic and oleophilic character of ODPA treated fibres due to $\mathrm{C}_{18} \mathrm{H}_{24}$ fatty chains brought by ODPA grafted at the fibre surfaces. It must be pointed out that pre-treatment in ethanol at $100{ }^{\circ} \mathrm{C}$ is likely to induce the removal of lipophilic and hydrophobic components (waxes) from the fibres (Acera Fernández et al. 2016). This was evidenced in the present study by the low contact angle with water $\left(81.3^{\circ}\right)$ and the very fast water sorption $(<4 \mathrm{~s})$ for ethanol pre-treated fibres. Nevertheless, the further functionalization of flax fibres with ODPA had a counterbalanced effect and even increased their

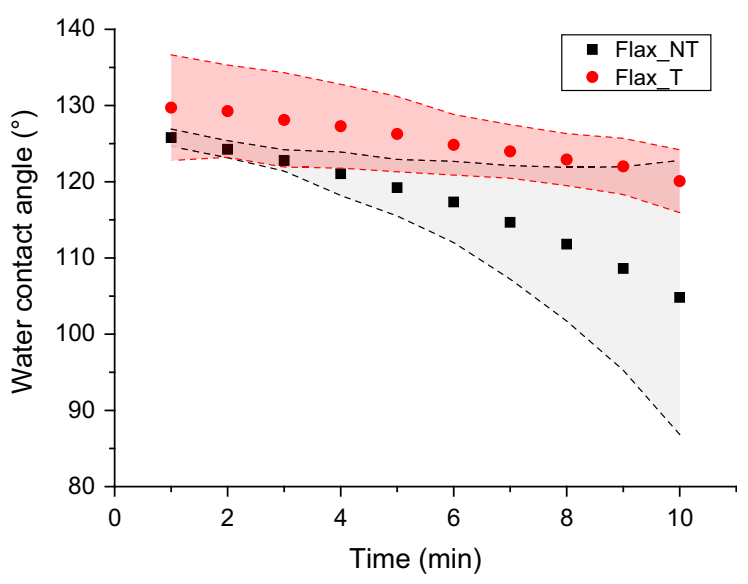

Fig. 5 Water contact angle overtime for untreated and ODPA treated flax fibres (dashed lines corresponds to standard deviation) 
hydrophobic and oleophilic character as compared to untreated fibres.

Effect of gamma radiation on cross-linking, crystallization and microstructure of biocomposites

\section{Gel fraction and localization of LDPE cross-linking}

The gel fraction was determined using Soxhlet extraction with boiling xylene in order to quantify the insoluble fraction of LDPE that would originate from LDPE cross-linking after gamma irradiation (Fig. 6). Measurements show that the gel fractions of the non-irradiated biocomposites were almost zero for both untreated and ODPA treated fibres, attesting that no self-cross-linking of LDPE chains or cross-linking with ODPA occurred during melt processing (i.e. extrusion and injection) of the composites. On the other hand, gamma radiation generates an average gel fraction of $22.0 \pm 6.2 \mathrm{wt} \%$ for the composite containing untreated fibres (LDPE/Flax_NT_I). The average gel fraction was similar $(22.0 \pm 1.6 \mathrm{wt} \%)$ for neat LDPE irradiated in the same conditions attesting that no specific interactions and cross-linking between LDPE and untreated flax fibres occurred upon gamma irradiation. It should be pointed out that the gel fraction of LDPE is greatly influenced by radiation conditions (Alvarez and Perez 2013), i.e. atmosphere and irradiation dose and type (e-beam or gamma), but

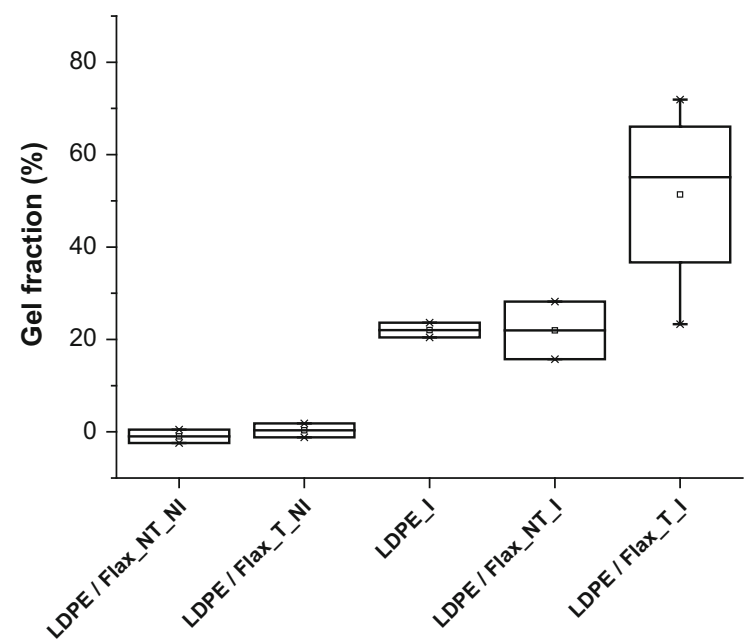

Fig. 6 Gel fraction (\%) box-plots of non-irradiated biocomposites, and irradiated neat LDPE and biocomposites also by the LDPE grade. Indeed, Alvarez and Perez (2013) found a gel fraction $<5 \%$ for LDPE irradiated at $30 \mathrm{kGy}$ under $21 \%$ oxygen. Bee et al. (2014) measured a gel fraction of $50 \%$ for e-beam irradiated LDPE at $20 \mathrm{kGy}$ in room conditions. A gel fraction of 3.8\% was found by Ferreto et al. (2012) after gamma radiation of LLDPE at $10 \mathrm{kGy}$ in room conditions. Considering the low radiation dose (10 kGy) used in our study, the measured gel fraction $(22 \mathrm{wt} \%)$ for neat LDPE is quite high.

Interestingly, irradiation of biocomposites containing ODPA treated fibres induces much higher average gel fraction, i.e. $51.4 \pm 14.7 \mathrm{wt} \%$. On a basis of 20 wt $\%$ ODPA treated fibres and $80 \mathrm{wt} \%$ LDPE matrix within the composite, it means that the ODPA flax fibres reinforced composite contains $41 \mathrm{wt} \%$ and 39 wt $\%$ of cross-linked and non-crosslinked LDPE chains, respectively. Considering such high gel fraction, part of these cross-linked LDPE chains should be located at the fibre/matrix interface and cross-linked with alkyl chain of ODPA, which promotes better interfacial adhesion. On the other hand, self-crosslinking of LDPE chains within the bulk of the matrix must also have occurred. The reason why the presence of ODPA at the flax fibre surfaces favours the crosslinking of LDPE chains is not fully elucidated. Sensitivity of ODPA $\mathrm{C}_{18} \mathrm{H}_{24}$ fatty chains towards gamma irradiation that would promote the formation of free radicals, and hence LDPE cross-linking, should be one of the explanations.

Gel fraction measurements were completed by SEM observations of the solid residues obtained after Soxhlet extraction. The SEM micrographs show the near absence of LDPE in the solid residue of nonirradiated biocomposites, i.e. only a fibrous network of elementary flax fibres is observed (Fig. 7a, b). On the contrary, cross-linked LDPE is observed on the fibres in the case of the irradiated biocomposites (Fig. 7c, d). The amount of LDPE appears much higher in the ODPA treated flax fibre composite (Fig. 7d), supporting that a part of the LDPE chains were cross-linked at the fibre/matrix interface due to the ODPA treatment.

These observations nicely confirm that the combined chemical and radiation-induced grafting approach developed in this work efficiently modifies interfacial interactions in LDPE/flax fibre biocomposites. A local and regio-selective cross-linking between LDPE chains and alkyl chain of ODPA grafted onto flax fibre surfaces occurred at the fibre/matrix 
Fig. 7 SEM micrographs of the solid residue after Soxhlet extraction in xylene for the different biocomposites: a LDPE/ flax_NT_NI; b LDPE/ flax_T_NI; c LDPE/ flax_NT_I, d LDPE/ flax_T_I
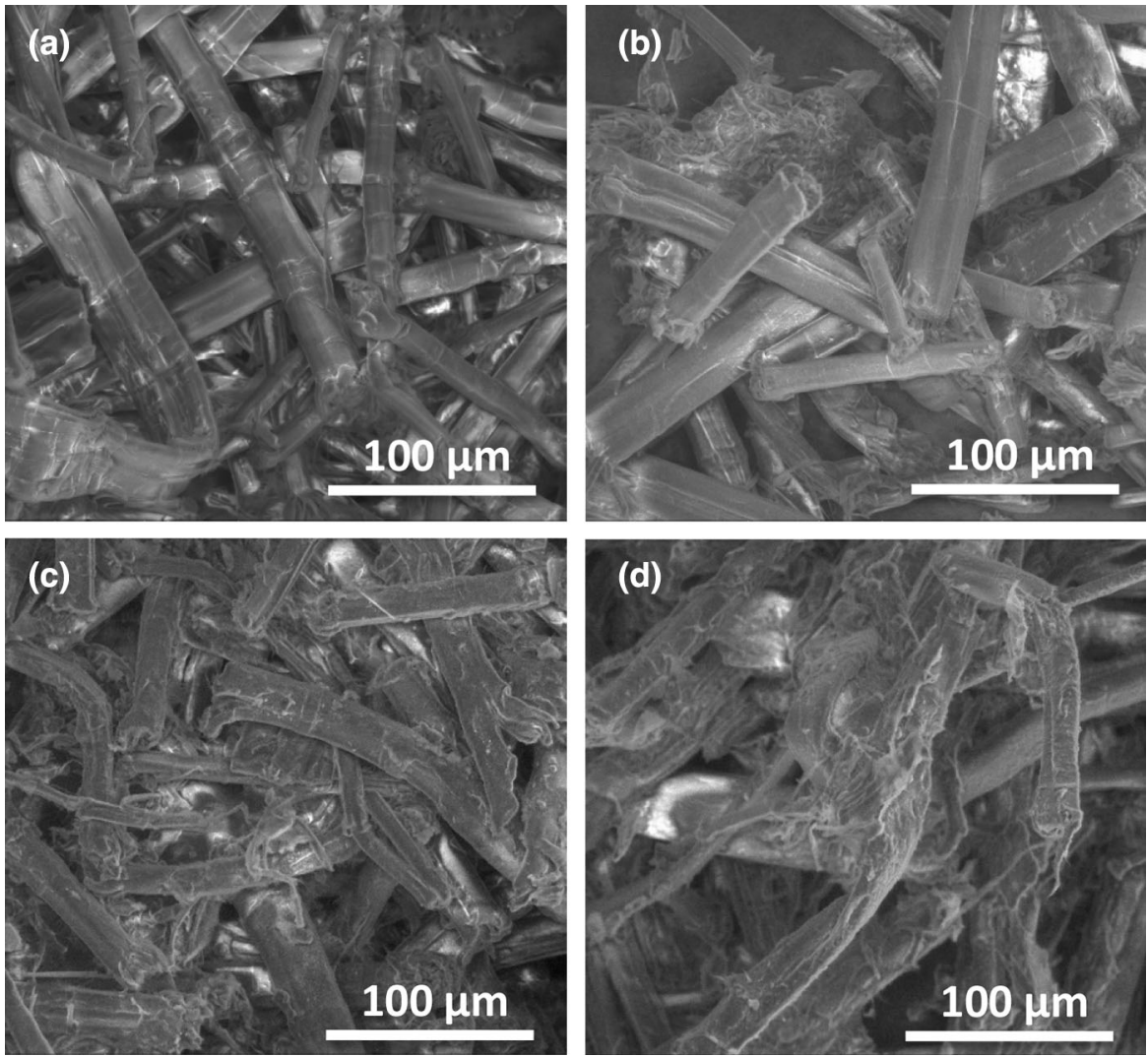

interface. Hence, covalent bonds were created on both fibre (grafted ODPA) and matrix (ODPA-PE crosslinks) sides.

\section{Crystallization of LDPE}

DSC analyses were carried out to study the effect of (un)treated flax fibres and gamma radiation on the crystallization of LDPE. Melting temperatures were similar for all composites (roughly $108^{\circ} \mathrm{C}$ ), meaning that there were no significant changes in the thermal stability of the crystals formed in the presence of (un)treated flax fibres or after gamma radiation. Crystallization temperatures $T_{c}$ were also very similar for all biocomposites (roughly $91{ }^{\circ} \mathrm{C}$ ) supporting that no noticeable nucleation effects related to the incorporation of (un)treated flax fibres occurred. Results in Fig. 8 show the degrees of crystallinity $\left(X_{c}\right)$ for the different composites and neat LDPE (with or without gamma radiation) based on the melting enthalpies measured by DSC during the first heating. The incorporation of flax fibres in the LDPE matrix is responsible for a decrease of $X_{c}$, from 41.0 to $36.0 \%$. On the other hand, we found that incorporating ODPA treated flax fibres results in a slight increase of $X_{c}$ up to

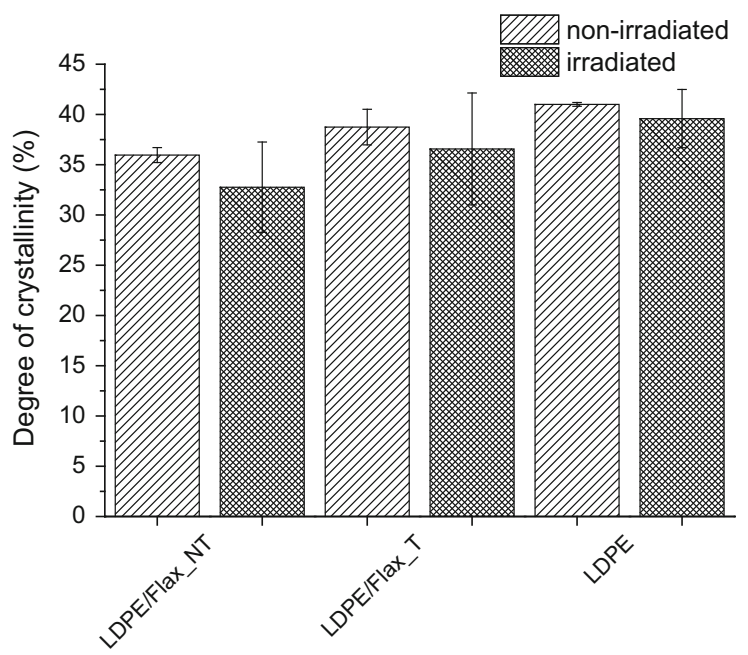

Fig. 8 Effect of ODPA treatment and gamma irradiation on the degree of crystallinity of neat LDPE matrix and LDPE in biocomposites 
$38.7 \%$ as compared to untreated fibres. Girones et al. (2017) studied the crystallization behaviour of polypropylene in the presence of various (ligno)cellulosic fibres and showed that lignin-poor fibres as flax fibres, can enhance crystallization kinetics and, depending on crystallization temperature (high degree of supercooling), could promote the formation of transcrystalline regions along the fibre surface. As pointed out by the authors, the chemical composition at the fibre surface plays a role on the crystallization behaviour of the matrix. Further isothermal crystallization analysis by DSC and polarized optical microscopy observations would be necessary to better depict the effect of flax fibres and ODPA treatment on the crystallization behaviour of LDPE.

For irradiated compounds, a slight decrease of the degree of crystallinity was observed, standard error being also higher. This can be ascribed to the presence of cross-linked LDPE chains within the irradiated materials. Indeed, the cross-linking of LDPE chains during gamma irradiation leads to the formation of a three-dimensional network of lower molecular mobility that should hinder the crystallization of LDPE, especially at high cross-linking degree. It should be noticed that the higher gel fraction $(51.4 \pm 14.7 \mathrm{wt} \%)$ measured for the composite containing ODPA treated flax fibres did not lead to more pronounced decrease of the degree of crystallinity.

Concluding, variations in LDPE crystallinity are relatively limited between the different composites $\left(X_{c}\right.$ varying from $32.8 \%$ for LDPE/Flax_NT_I to $38.7 \%$ for LDPE/Flax_T_NI) and are thus not expected to induce significant variations in their mechanical behaviour. At the macromolecular level, cross-linking at the fibre/matrix interface and within the bulk of the matrix induced by gamma irradiation and enhanced by the presence of ODPA is the main phenomenon that should influence the interfacial adhesion and tensile mechanical behaviour of the composites.

\section{Dispersion state of the fibres and size and shape distribution}

Microstructural analysis was conducted to investigate the effect of fibre treatment and gamma irradiation on the fibre size and shape distribution within the biocomposites after extrusion and injection. An example of a $3.5 \mathrm{~mm} \times 3.5 \mathrm{~mm}$ cartography obtained by SEM on polished tensile specimen is shown in
Fig. 9. Generally speaking, flax fibres were well dispersed and individualized into elementary fibres for all biocomposites with the presence of fibre bundles and particles as defined by Le Moigne et al. (2011). As described by Bourmaud et al. (2013) for PP/flax composites, several fibres are also perpendicular to the field of observation due to the well-known fountain flow effect occurring during injection moulding. This flow effect influences fibre orientation according to the distance from the mould wall and in relation with the flow type, either extensional flow at the centre or shear flow at the wall. This was observed at the two (left and right) edges of the cartographies, and perpendicular fibres were thus seen in their cross-section as small particles with low aspect ratio. Although this phenomenon could influence the fibre size and shape analysis, it was similar for all biocomposites, i.e. a thin layer of about $250 \mu \mathrm{m}$ at the edges of the samples with predominantly perpendicular oriented fibres.

When comparing the fibre aspect ratio distributions for the different biocomposites (Fig. 10), it is found that median aspect ratios range from 2.7 to 3.8 and from 2.9 to 4.4 for number and surface averaged distributions, respectively. It can be noticed that the

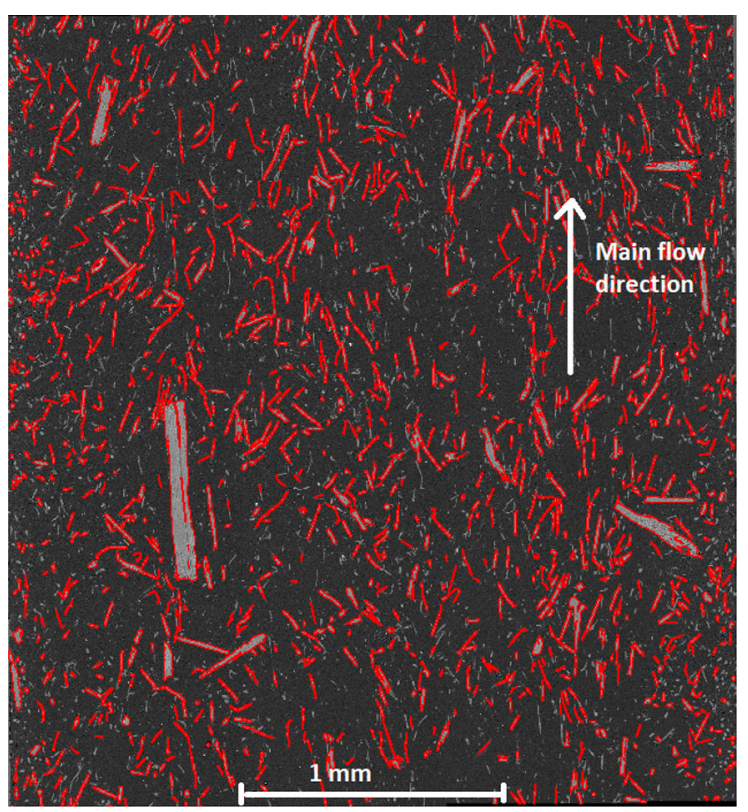

Fig. 9 An example of $3.5 \mathrm{~mm} \times 3.5 \mathrm{~mm}$ cartography obtained for the LDPE/flax_NT_NI biocomposites by SEM. Red lines correspond to automatic detection of the outlines of the fibres further used for image analysis and determination of Ferret diameters 
reference biocomposite (LDPE/flax_NT_NI) has in fact higher aspect ratio (this is even more pronounced when considering the distribution weighted in surface, see Fig. 10). ODPA and gamma irradiation treatments have thus an effect on the resulting fibre shape distributions after injection. As studied by Castellani et al. (2016), fibre breakage mechanisms during processing are complex and intimately related to the biochemical composition and the structure of natural fibres. It could thus be hypothesized that ODPA treatment and gamma irradiation induce biochemical and structural modifications and possibly degrade the fibres (Acera Fernández et al. 2016; Le Moigne et al. 2017). This modifies fibre breakage mechanisms during compounding and injection moulding and leads to lower fibres aspect ratio. Such microstructural modification should influence the mechanical behaviour of the biocomposites, in particular in the load transfer efficiency and hence their stiffness and strength properties. The higher the aspect ratio, the greater the load transfer and the higher should be the stiffness and strength.

Mechanical behaviour and interfacial adhesion of biocomposites

\section{Uniaxial tensile properties}

All biocomposites had a visco-elasto-plastic behaviour. Figure 11 shows the tensile behaviour (11a, b) and main tensile properties of the different composites, i.e. Young's modulus (11c), ultimate strength (11d), strain at break (11e) and work of rupture (11f).
As expected, the incorporation of $20 \mathrm{wt} \%$ [14 vol\% based on density calculation with flax fibre density of $1.4 \mathrm{~g} / \mathrm{cm}^{3}$ (Le Gall et al. 2018)] flax fibres within LDPE matrix (LDPE/Flax_NT_NI) has a strong reinforcing effect with median Young's modulus and ultimate strength values of $723 \mathrm{MPa}$ and $18.3 \mathrm{MPa}$, respectively, against $118 \mathrm{MPa}$ and $10.3 \mathrm{MPa}$, respectively, for the neat LDPE. On the other hand, strain at break and work of rupture are greatly decreased, i.e. $87.5 \%$ and $14.8 \mathrm{~J}$ respectively for neat LDPE, against $11.7 \%$ and $0.78 \mathrm{~J}$ respectively for the untreated flax fibre composite. This is the direct consequence of the presence of fibres which act as defaults initiating breakage. It was also found that gamma irradiation had no reinforcing effect on neat LDPE with similar Young's modulus and ultimate strength (108.0 MPa and 9.6 MPa respectively for LDPE_I), but noticeable lower strain at break and work of rupture $(72.4 \%$ and $11.1 \mathrm{~J}$ respectively for LDPE_I). When considering the neat LDPE matrix, incorporating flax fibres has thus a pronounced reinforcing effect but reduces its ductility (lower work of rupture) due to the rigid fibre network [note that based on the calculated fibre volume fraction and measured aspect ratios, the fibre concentration corresponds to the semi-dilute regime (Le Moigne et al. 2013)], and higher probability of crack initiation at high strain. This is also related to low interfacial adhesion with untreated flax fibres as seen in Fig. 12a, with many fibres pull-out and the presence of interfacial gaps between the fibres and the matrix. Gamma irradiation also decreases the ductility of neat LDPE matrix.

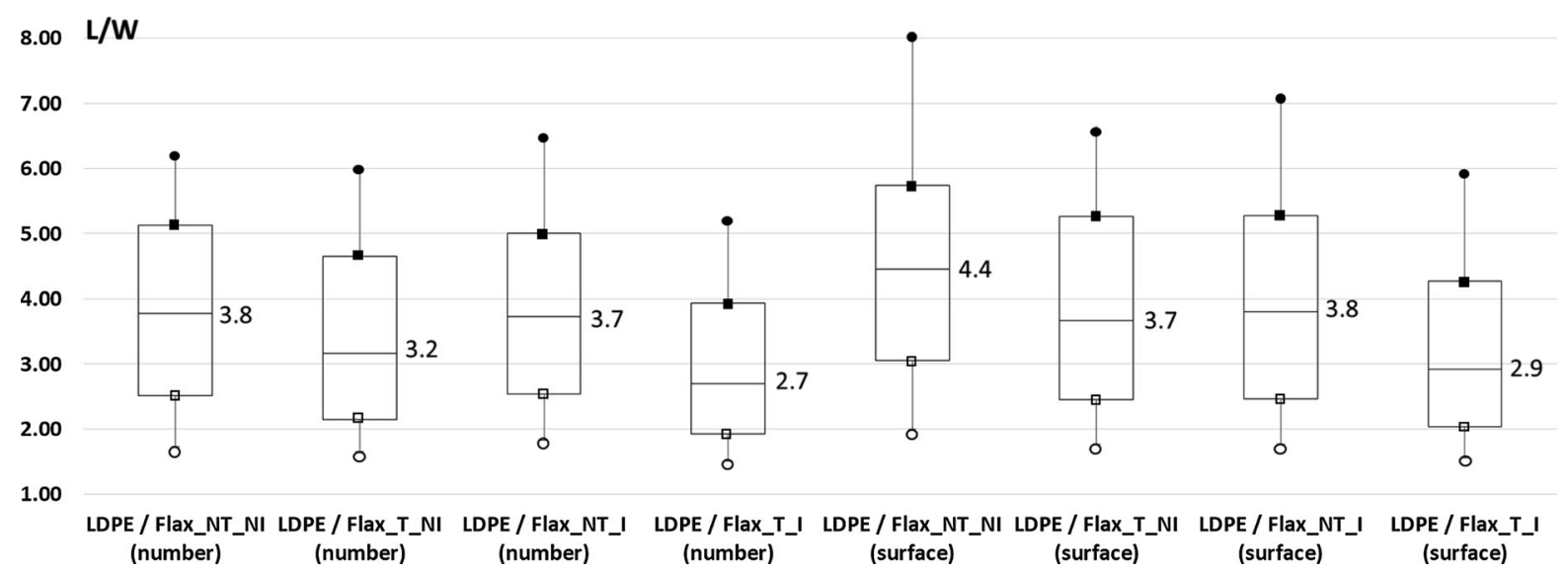

Fig. 10 Box plots of fibre aspect ratio distributions weighted in number and in surface for the different biocomposites 

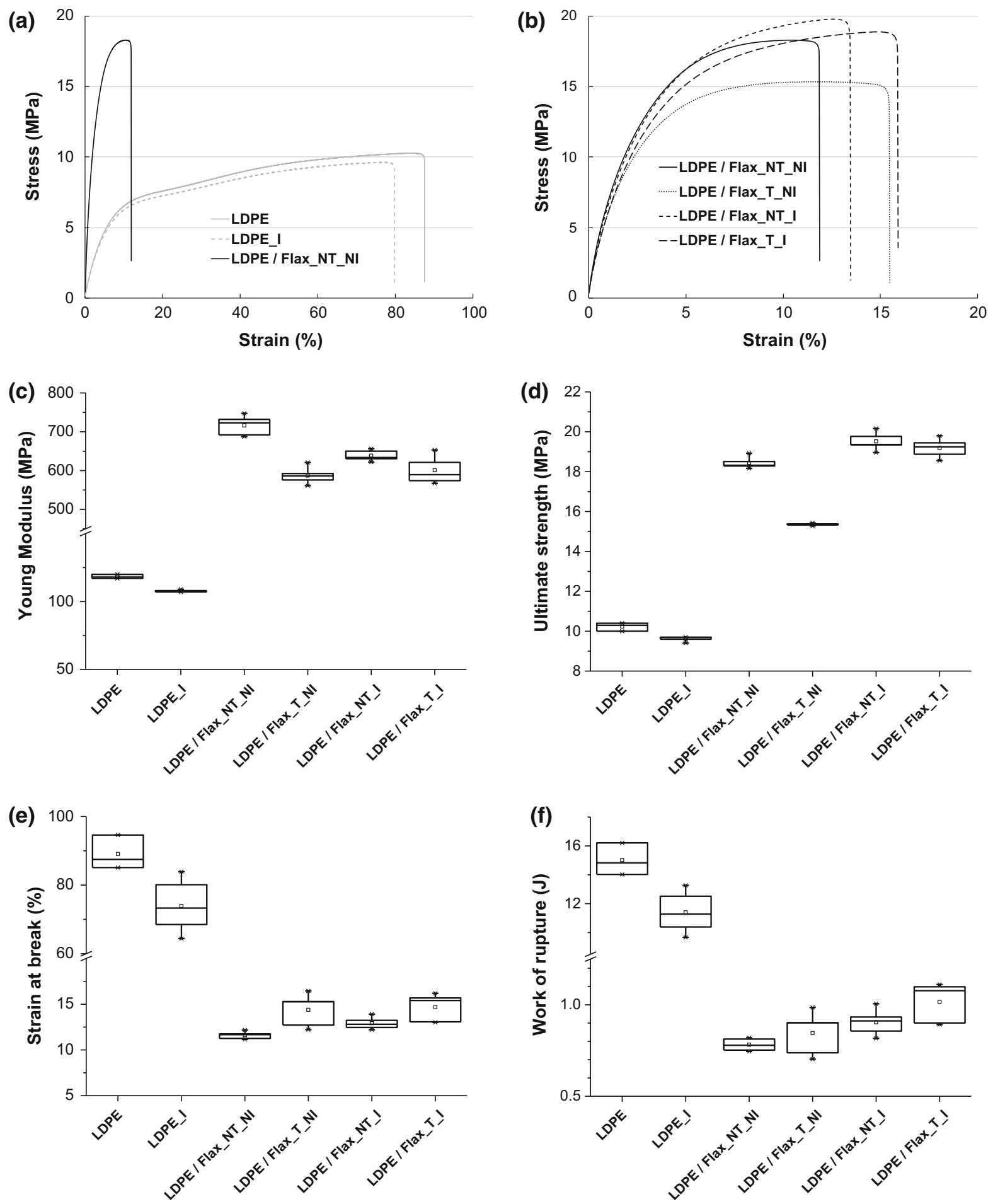

Fig. 11 Effect of flax fibres, ODPA treatment and gamma irradiation on tensile behaviour of neat LDPE and LDPE/flax biocomposites $(\mathbf{a}, \mathbf{b})$ and their tensile properties: c Young's modulus, $\mathbf{d}$ Ultimate strength, e strain at break, $\mathbf{f}$ work of rupture 
When comparing the different biocomposites and the effect of treatments, the observed trend for the Young's modulus is in accordance with the aspect ratio distribution weighted in number and surface (Note that the crystallinity rate is quite similar for all composites and should not induce variations in the mechanical behaviour). The greater is the aspect ratio, the higher is the Young modulus. In the absence of irradiation, the lower fibre aspect ratio obtained with ODPA treatment leads to a composite with low ultimate strength. Simultaneously, the increase of ultimate strain values (i.e. higher ductility) and work of rupture suggests that ODPA treatment improves the interfacial adhesion between flax and matrix probably due to a better affinity between LDPE chains and $\mathrm{C}_{18} \mathrm{H}_{24}$ fatty chains beared by ODPA. Interestingly, gamma irradiation leads to an enhancement of the ultimate properties of both untreated (LDPE/ Flax_NT_I) and ODPA treated (LDPE/Flax_T_I) biocomposites (even if the fibre aspect ratio distribution is lower); ultimate strength, strain at break and work of rupture being greatly increased as compared to the non-irradiated biocomposites (up to 19.2 $\mathrm{MPa}$, $14.3 \%$ and $1.08 \mathrm{~J}$, respectively, for LDPE/Flax_T_I).
It is also worth mentioning that the combined ODPA and gamma radiation treatment (LDPE/Flax_T_I) leads to equivalent ultimate strength but greater ductility with higher work of rupture, supporting that higher interfacial adhesion was achieved due to the local ODPA-LDPE cross-linking at the fibre/matrix interface. This is also supported by SEM observations revealing more broken fibres which were better embedded within the matrix for this composite (Fig. 12d).

\section{Failure mechanisms at the fibre/matrix interface}

To better depict the effect of the ODPA and gamma radiation treatments on the interfacial adhesion between flax fibres and LDPE, micro-mechanical tensile tests conducted in-situ in the SEM chamber were performed on the different biocomposites. At the micro-scale, ODPA and irradiation treatments clearly lead to higher peak force and elongation at break. Based on the force-elongation curves obtained with the micro-mechanical tensile tests (Fig. 13a), the LDPE/Flax_NT_NI biocomposite breaks at $93 \mathrm{~N}$ with maximum elongation at break of $0.84 \mathrm{~mm}$, while all
Fig. 12 Failure surfaces observed by SEM after uniaxial tensile tests for a LDPE/flax_NT_NI, b LDPE/flax_T_NI, c LDPE/flax_NT_I, d LDPE/flax_T_I
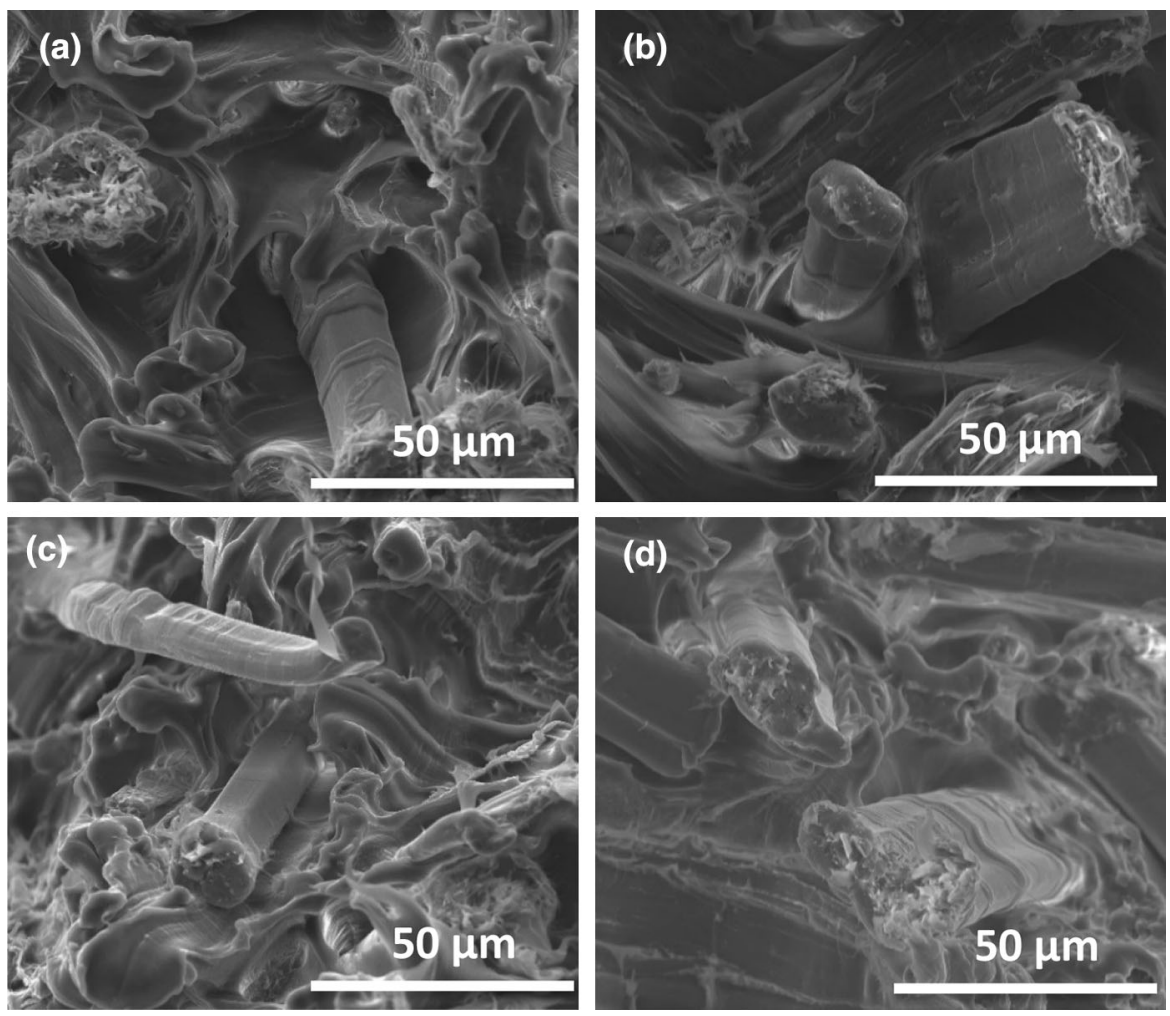
other biocomposites break at higher loading forces (up to $117 \mathrm{~N}$ for LDPE/Flax_T_I) and present much higher elongation at break (up to $1.35 \mathrm{~mm}$ for LDPE/ Flax_T_I). This shows higher strength and a more ductile behaviour, hence confirming uniaxial tensile results. This also results in higher work of rupture that can be correlated with work of rupture measured from uniaxial tensile tests (Fig. 13b). The micro-tensile tests performed in the SEM can thus provide meaningful results on the reinforcement potential induced by a specific treatment at the fibre/matrix interface.

Besides, micro-mechanical tensile SEM experiments allow studying the failure mechanisms by insitu visualizations of the crack propagation in notched samples. LDPE/Flax_NT_NI (Video 1) and LDPE/ Flax_T_I biocomposites (Video 2) present very different failure behaviours. In the case of the reference biocomposite, the crack occurs by stretching and tearing of the matrix while debonding of the fibres occurs at the interface, which is characteristic of an adhesive interfacial failure (Video 1). In contrast, the ODPA treated and irradiated biocomposite shows a more cohesive interfacial failure characterized by a breaking of the fibres that remain bonded to the matrix (Video 2).

Mechanical behaviour studied at the macro-scale (uniaxial tensile) and the micro-scale (in-situ tensile SEM) thus evidences that higher interfacial adhesion was achieved with the ODPA and irradiation treatments, especially when combined, leading to higher ultimate properties. Even if these treatments slightly reduce the aspect ratio of the fibres within the

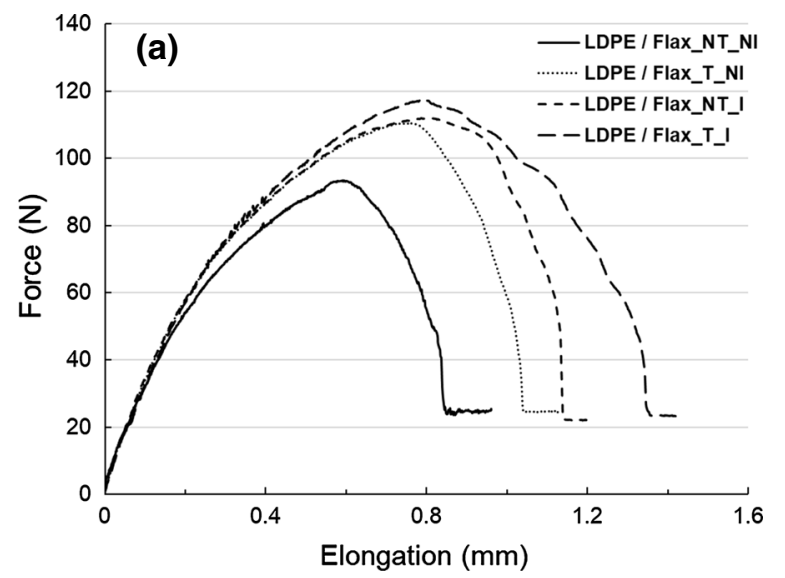

composites, interfacial adhesion is sufficiently improved to counterbalance the detrimental effect of decreased aspect ratio and even improve biocomposites strength.

\section{Conclusions}

The goal of this work was to improve the interfacial adhesion between flax fibres and a non-polar and nonreactive matrix, i.e. low density polyethylene (LDPE), so as to enhance the mechanical performances of LDPE/flax fibre biocomposites. As described in Fig. 14, flax fibres were first pre-treated with ethanol, then treated with octadecylphosphonic acid (ODPA). It was found that ODPA molecules were effectively grafted onto flax fibres at a content of roughly $2.5 \%$ and mostly localized at the fibre surfaces. Moreover, contact angle measurements with water and diiodomethane as well as water sorption kinetics gave evidence for increased hydrophobic and oleophilic character of ODPA treated fibres. Compounds of LDPE/flax fibres prepared by melt extrusion were then submitted to gamma radiation at a dose of $10 \mathrm{kGy}$ before injection moulding to obtain the composite specimens. Based on Soxhlet extraction experiments, it was shown that LDPE gel fraction was greatly increased in the case of the OPDA treated and irradiated biocomposite (up to $51.4 \mathrm{wt} \%$ ). SEM observations of the solid residues after Soxhlet extraction revealed that LDPE cross-linking rate induced by gamma radiation was locally enhanced at

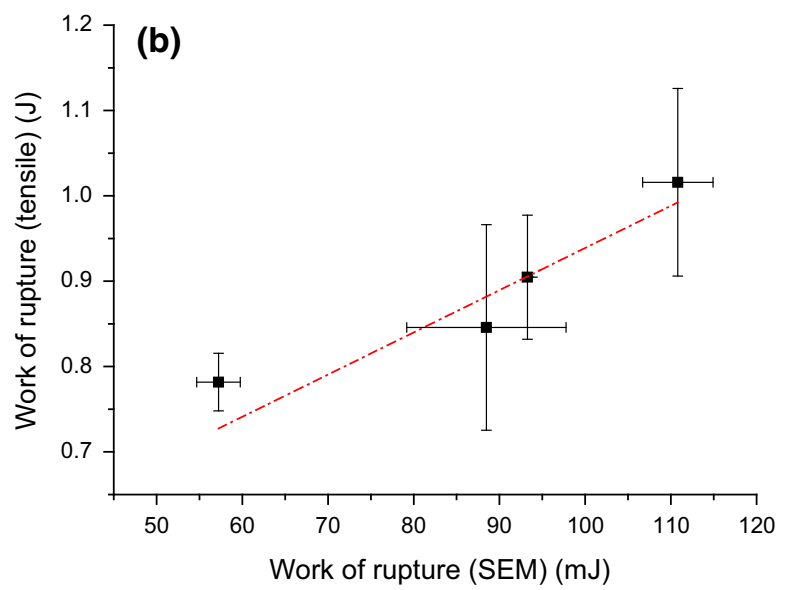

Fig. 13 a Loading force versus elongation curves of in-situ tensile SEM tests for notched specimens, b correlation between work of rupture measured by uniaxial tensile tests and in-situ tensile SEM 


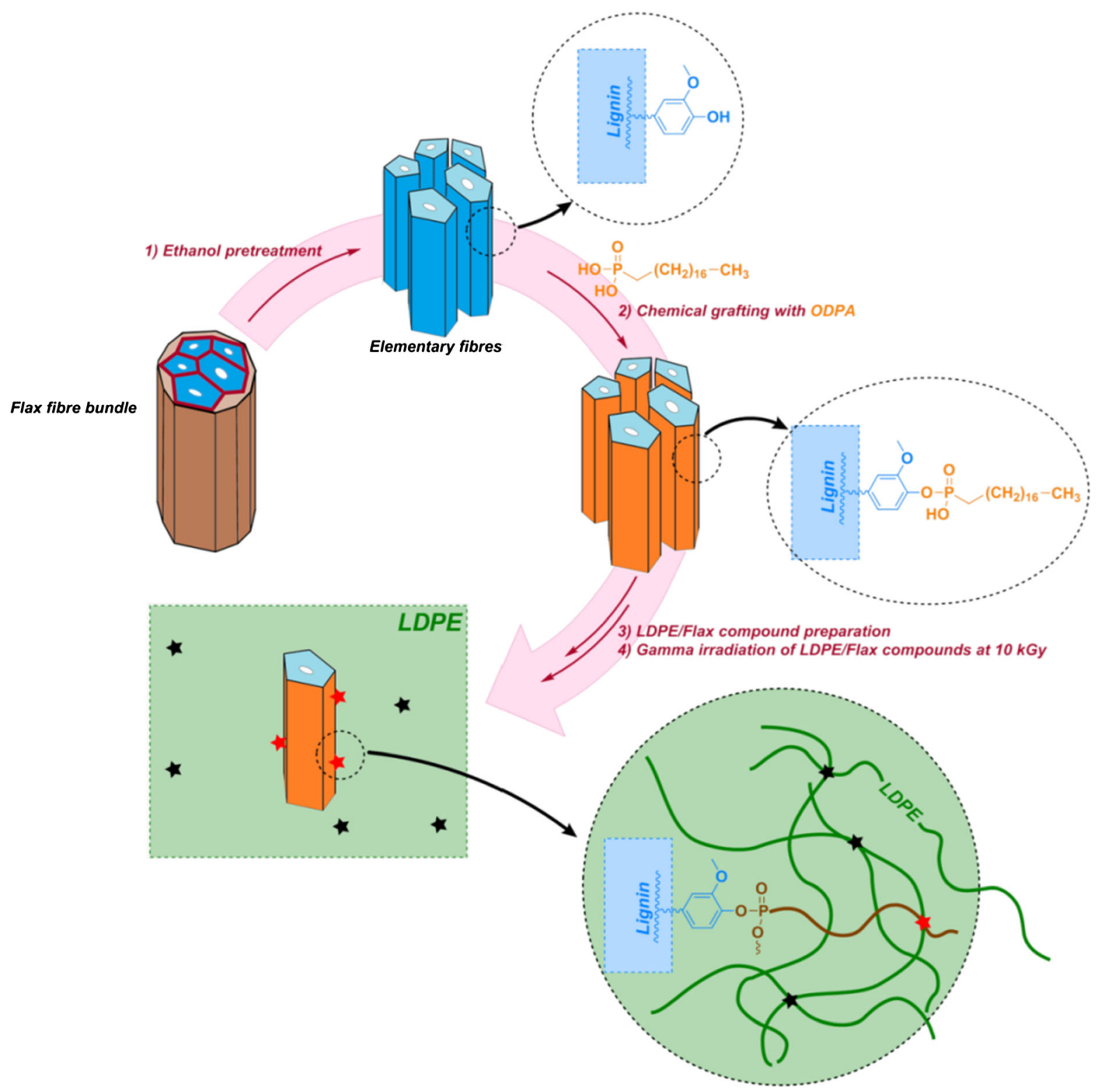

Fig. 14 Scheme of the combined chemical and radiationinduced grafting approach to improve the interfacial adhesion between flax fibres and LDPE: (1) ethanol pre-treatment, (2) chemical grafting of ODPA onto flax fibre surfaces, (3) and (4)

the fibre/matrix interface for this biocomposite, suggesting that regio-selective cross-linking occurred between LDPE chains and the alkyl chain of ODPA grafted on flax fibre surfaces. Interfacial adhesion has been sufficiently improved by this combined chemical and radiation-induced grafting approach to improve significantly the ultimate properties of the biocomposites (up to $40 \%$ increase in the work of rupture). compounding of LDPE/flax fibre biocomposites followed by gamma radiation inducing LDPE self-cross-linking (black stars) and ODPA-LDPE radical reaction (red stars)

These results were supported by in-situ micro-mechanical tensile SEM experiments, which revealed more cohesive interfacial failure and higher work of rupture nicely correlated with work of rupture measured by uniaxial tensile tests. Our results open interesting perspectives for the development of performing interfaces within biocomposites based on non-polar and non-reactive matrices and reinforced 
with natural fibres. Besides, further improvement of the treatment process being a key for industry, recent advances in electron-induced reactive processing could be considered.

Acknowledgments The authors acknowledge IMT Mines Alès for supporting the FERIA Project (Prix André Lefebvre). We are grateful to the research institute on catalysis and the environment of Lyon (IRCELYON) for the solid state ${ }^{31}$ P RMN analysis and to Sophie ROUIF (Ionisos, Dagneux, France) for gamma radiation treatment of biocomposites. The authors would like to thank Jean-Claude ROUX (IMT Mines Alès) for SEM experiments, and Hazem BEN ABI ZID, Romain RAVEL, Benjamin GALLARD, Alain DIAZ, Loïc DUMAZERT and Alexandre CHERON (IMT Mines Alès) for their scientific and technical support.

\section{References}

Abdennadher A (2015) Injection moulding of natural fibres reinforced-polypropylene: process, microstructure and properties. Mines ParisTech, New York

Acera Fernández J, Le Moigne N, Caro-Bretelle AS et al (2016) Role of flax cell wall components on the microstructure and transverse mechanical behaviour of flax fabrics reinforced epoxy biocomposites. Ind Crops Prod 85:93-108. https:// doi.org/10.1016/j.indcrop.2016.02.047

Alvarez VA, Perez CJ (2013) Gamma irradiated LDPE in presence of oxygen. Part I. Non-isothermal crystallization. Thermochim Acta 570:64-73. https://doi.org/10.1016/j. tca.2013.07.026

Bay RS, Tucker CL (1992) Fiber orientation in simple injection moldings. Part I: Theory and numerical methods. Polym Compos 13:317-331. https://doi.org/10.1002/pc. 750130409

Bee S-T, Ratnam CT, Sin LT et al (2014) Effects of electron beam irradiation on the structural properties of polylactic acid/polyethylene blends. Nucl Instrum Methods Phys Res Sect B Beam Interact Mater Atoms 334:18-27. https://doi. org/10.1016/j.nimb.2014.04.024

Bourmaud A, Ausias G, Lebrun G et al (2013) Observation of the structure of a composite polypropylene/flax and damage mechanisms under stress. Ind Crops Prod 43:225-236. https://doi.org/10.1016/j.indcrop.2012.07.030

Bourmaud A, Beaugrand J, Shah DU et al (2018) Towards the design of high-performance plant fibre composites. Prog Mater Sci 97:347-408. https://doi.org/10.1016/j.pmatsci. 2018.05.005

Castellani R, Di Giuseppe E, Beaugrand J et al (2016) Lignocellulosic fiber breakage in a molten polymer. Part 1 . Qualitative analysis using rheo-optical observations. Compos Part A Appl Sci Manuf 91:229-237. https://doi. org/10.1016/j.compositesa.2016.10.015

Coroller G, Lefeuvre A, Le Duigou A et al (2013) Effect of flax fibres individualisation on tensile failure of flax/epoxy unidirectional composite. Compos Part A Appl Sci Manuf 51:62-70. https://doi.org/10.1016/j.compositesa.2013.03. 018
Dogan SD, Tayfun U, Dogan M (2016) New route for modifying cellulosic fibres with fatty acids and its application to polyethylene/jute fibre composites. J Compos Mater 50:2477-2485. 0021998315604706

https://doi.org/10.1177/

Dorez G, Otazaghine B, Taguet A et al (2014) Use of Py-GC/MS and PCFC to characterize the surface modification of flax fibres. J Anal Appl Pyrolysis 105:122-130. https://doi.org/ 10.1016/j.jaap.2013.10.011

Ferreto HFR, Oliveira ACF, Lima LFCP et al (2012) Thermal, tensile and rheological properties of linear low density polyethylene (LLDPE) irradiated by gamma-ray in different atmospheres. Radiat Phys Chem 81:958-961. https:// doi.org/10.1016/j.radphyschem.2012.02.003

Freire CSR, Silvestre AJD, Neto CP et al (2006) Controlled heterogeneous modification of cellulose fibers with fatty acids: effect of reaction conditions on the extent of esterification and fiber properties. J Appl Polym Sci 100:1093-1102. https://doi.org/10.1002/app.23454

Freire CSR, Silvestre AJD, Neto CP et al (2008) Composites based on acylated cellulose fibers and low-density polyethylene: effect of the fiber content, degree of substitution and fatty acid chain length on final properties. Compos Sci Technol 68:3358-3364. https://doi.org/10.1016/j. compscitech.2008.09.008

Girones J, Vo LTT, Haudin J-M et al (2017) Crystallization of polypropylene in the presence of biomass-based fillers of different compositions. Polymer 127:220-231. https://doi. org/10.1016/j.polymer.2017.09.006

Hajj R, El Hage R, Sonnier R et al (2018) Grafting of phosphorus flame retardants on flax fabrics: comparison between two routes. Polym Degrad Stab 147:25-34. https://doi.org/10.1016/j.polymdegradstab.2017.11.006

Holland GP, Sharma R, Agola JO et al (2007) NMR characterization of phosphonic acid capped $\mathrm{SnO}_{2}$ nanoparticles. Chem Mater 19:2519-2526. https://doi.org/10.1021/ $\mathrm{cm} 062821 \mathrm{u}$

Kazayawoko M, Balatinecz JJ, Matuana LM (1999) Surface modification and adhesion mechanisms in woodfiberpolypropylene composites. J Mater Sci 34:6189-6199. https://doi.org/10.1023/A:1004790409158

Krishnaswamy RK, Yang Q (2007) Influence of phase segregation on the mechanical properties of binary blends of polyethylenes that differ considerably in molecular weight. Polymer 48:5348-5354. https://doi.org/10.1016/j.polymer. 2007.07.018

Le Duigou A, Bourmaud A, Balnois E et al (2012) Improving the interfacial properties between flax fibres and PLLA by a water fibre treatment and drying cycle. Ind Crops Prod 39:31-39. https://doi.org/10.1016/j.indcrop.2012.02.001

Le Duigou A, Merotte J, Bourmaud A et al (2017) Hygroscopic expansion: a key point to describe natural fibre/polymer matrix interface bond strength. Compos Sci Technol 151:228-233. https://doi.org/10.1016/j.compscitech.2017. 08.028

Le Gall M, Davies P, Martin N, Baley C (2018) Recommended flax fibre density values for composite property predictions. Ind Crops Prod 114:52-58. https://doi.org/10.1016/j. indcrop.2018.01.065

Le Moigne N, Van Den Oever M, Budtova T (2011) A statistical analysis of fibre size and shape distribution after 
compounding in composites reinforced by natural fibres. Compos Part A Appl Sci Manuf 42:1542-1550. https://doi. org/10.1016/j.compositesa.2011.07.012

Le Moigne N, Van Den Oever M, Budtova T (2013) Dynamic and capillary shear rheology of natural fiber-reinforced composites. Polym Eng Sci. https://doi.org/10.1002/pen. 23521

Le Moigne N, Longerey M, Taulemesse JM et al (2014) Study of the interface in natural fibres reinforced poly(lactic acid) biocomposites modified by optimized organosilane treatments. Ind Crops Prod 52:481-494. https://doi.org/10. 1016/j.indcrop.2013.11.022

Le Moigne N, Sonnier R, El Hage R, Rouif S (2017) Radiationinduced modifications in natural fibres and their biocomposites: opportunities for controlled physico-chemical modification pathways? Ind Crops Prod 109:199-213. https://doi.org/10.1016/j.indcrop.2017.08.027

Le Moigne N, Otazaghine B, Corn S et al (2018a) Interfaces in natural fibre reinforced composites: definitions and roles. In: Navard P (ed) Surfaces and interfaces in natural fibre reinforced composites. Springer, Berlin, pp 23-34

Le Moigne N, Otazaghine B, Corn S et al (2018b) Introduction on natural fibre structure: from the molecular to the macrostructural level. In: Navard P (ed) Surfaces and interfaces in natural fibre reinforced composites. Springer, Berlin, pp 1-22

Le Moigne N, Otazaghine B, Corn S et al (2018c) Modification of the interface/interphase in natural fibre reinforced composites: treatments and processes. In: Navard P (ed) Surfaces and interfaces in natural fibre reinforced composites. Springer, Berlin, pp 35-70

Lu J, Sue H-J (2002) Morphology and mechanical properties of blown films of a low-density polyethylene/linear lowdensity polyethylene blend. J Polym Sci Part B Polym Phys 40:507-518. https://doi.org/10.1002/polb.10115

Marques G, del Río JC, Gutiérrez A (2010) Lipophilic extractives from several nonwoody lignocellulosic crops (flax, hemp, sisal, abaca) and their fate during alkaline pulping and TCF/ECF bleaching. Bioresour Technol 101:260-267. https://doi.org/10.1016/j.biortech.2009.08.036

Morvan C, Andème-Onzighi C, Girault R et al (2003) Building flax fibres: more than one brick in the walls. Plant Physiol Biochem 41:935-944. https://doi.org/10.1016/j.plaphy. 2003.07.001
Müssig J (ed) (2010) Industrial applications of natural fibres: structure, properties and technical applications. Wiley, Chichester

Poltimäe T, Tarasova E, Krumme A et al (2009) Behaviour of the very-low-temperature crystallization peak of linear low-density polyethylene. Proc Est Acad Sci 58:58. https:// doi.org/10.3176/proc.2009.1.10

Réquilé S, Le Duigou A, Bourmaud A, Baley C (2019) Interfacial properties of hemp fiber/epoxy system measured by microdroplet test: effect of relative humidity. Compos Sci Technol 181:107694. https://doi.org/10.1016/j. compscitech.2019.107694

Sonnier R, Otazaghine B, Viretto A et al (2015) Improving the flame retardancy of flax fabrics by radiation grafting of phosphorus compounds. Eur Polym J 68:313-325. https:// doi.org/10.1016/j.eurpolymj.2015.05.005

Torres FG, Cubillas ML (2005) Study of the interfacial properties of natural fibre reinforced polyethylene. Polym Test 24:694-698. https://doi.org/10.1016/j.polymertesting. 2005.05.004

Ungar G (1981) Radiation effects in polyethylene and $n$-alkanes. J Mater Sci 16:2635-2656. https://doi.org/10.1007/ BF02402826

Xie Y, Hill CS, Xiao Z et al (2010) Silane coupling agents used for natural fiber/polymer composites: a review. Compos Part A Appl Sci Manuf 41:806-819. https://doi.org/10. 1016/j.compositesa.2010.03.005

Yah WO, Takahara A, Lvov YM (2012) Selective modification of halloysite lumen with octadecylphosphonic acid: new inorganic tubular micelle. J Am Chem Soc 134:1853-1859. https://doi.org/10.1021/ja210258y

Yan L, Chouw N, Jayaraman K (2014) Flax fibre and its composites-a review. Compos Part B Eng 56:296-317. https://doi.org/10.1016/j.compositesb.2013.08.014

Zafeiropoulos N, Baillie C, Hodgkinson J (2002) Engineering and characterisation of the interface in flax fibre/ polypropylene composite materials. Part II. The effect of surface treatments on the interface. Compos Part A Appl Sci Manuf 33:1185-1190. https://doi.org/10.1016/S1359835X(02)00088-X 